\title{
Birth weight, subsequent growth, and cholesterol metabolism in children 8-12 years old born preterm
}

\author{
M Mortaz, M S Fewtrell, T J Cole, A Lucas
}

\begin{abstract}
Aims-To test the hypothesis that plasma lipids, lipoproteins, and markers of cholesterol biosynthesis (lathosterol) and absorption efficiency (campesterol) in children aged 8-12 years are related to birth size and subsequent growth.

Methods-A total of 412 girls and boys weighing less than $1850 \mathrm{~g}$ at birth were studied. Birth weight, gestation, and weight at 18 months were recorded and followed up at 8-12 years. Plasma total cholesterol, low density lipoprotein cholesterol, high density lipoprotein cholesterol, apolipoprotein A1, apolipoprotein B, triacylglycerol, lathosterol, and campesterol were measured.

Results-Birth weight for gestational age was positively related to plasma campesterol, and remained so after adjusting for current body size or fatness. Birth weight was negatively related to current plasma lathosterol but only after adjusting for current body size or fatness. For both lathosterol and campesterol the significant relation with birth size adjusted for current size indicates that the change in size between these points (postnatal upward centile crossing) was influential. These relations were absent for total cholesterol, lipoproteins, apolipoproteins, and triacylglycerol.

Conclusion-Preterm children who were smaller for gestational age at birth had lower predicted cholesterol absorption efficiency 8-12 years later. Among children of the same current size, predicted endogenous cholesterol synthesis was higher and cholesterol absorption efficiency lower in those who showed the greatest increase in weight centile between birth and follow up. This finding was not confined to children with the smallest birth weights for gestational age. We suggest that both fetal and childhood growth relate to programming of cholesterol metabolism in children born preterm.

(Arch Dis Child 2001;84:212-217)
\end{abstract}

Keywords: birth weight; growth; cholesterol metabolism; preterm

Dr Mortaz, MRC Childhood Nutrition Centre, Institute of Child Health, 30 Guilford Street, London WC1N 1EH, UK.

m.mortaz@ich.ucl.ac.uk

Accepted 23 August 2000 cholesterol in later life. For example, overfeeding during early postnatal life in rats has been shown to have a long term influence on plasma cholesterol and insulin in adulthood. ${ }^{3}$ Our recent studies in rats have also shown that dietary protein restriction during gestation and lactation can alter plasma cholesterol and the activity of the rate limiting enzyme of cholesterol synthesis in adult offspring rats, ${ }^{45}$ suggesting that in rats the activity of the key enzyme in the cholesterol synthesis pathway may be programmed by maternal nutrition during a critical early period. Infant nutrition has also been shown to have a lifetime effect on cholesterol metabolism and, indeed, development of atherosclerosis in non-human primates. ${ }^{6}$

Corresponding evidence in humans is more limited and comes mainly from epidemiological studies relating small size or thinness at birth or during infancy with increased serum total cholesterol, low density lipoprotein (LDL) cholesterol, and apolipoprotein $\mathrm{B}^{7}$ and with other related risk factors for CVD such as high plasma clotting factors ${ }^{8}$ and impaired glucose tolerance ${ }^{9}$ in later life. Barker and colleagues suggested that nutritionally impaired growth, especially of the liver, during late gestation could result in permanent changes in cholesterol metabolism which persist until adult life. ${ }^{10}$ More recently, studies have attempted to investigate the influence of postnatal growth on later risk of $\mathrm{CVD}^{11}$ and suggested that this might interact with poor growth in utero to increase CVD risk.

However, there are many unanswered questions: what is the relative impact of pre- and postnatal nutrition on plasma lipids; what are the metabolic alterations in cholesterol metabolism which underlie changes of plasma lipids; and can these early influences be detected in childhood when atherosclerosis may originate? ${ }^{12}$

Measurement of cholesterol metabolism includes direct assessment of cholesterol synthesis and absorption, but this is expensive, time consuming, and difficult for population studies. However, plasma concentrations of lathosterol, a precursor of cholesterol, and campesterol, a plant sterol are indicators of whole body cholesterol synthesis and absorption, respectively, that can be measured by gas liquid chromatography-mass spectrometry (GC-MS). ${ }^{13-15}$ Plasma lathosterol and campesterol concentrations have been correlated positively with changes in overall cholesterol 
synthesis and absorption efficiency in many experimental and clinical conditions. ${ }^{15-17}$

To our knowledge neither size at birth nor growth in childhood have been studied in relation to later cholesterol synthesis and absorption. We have explored this in a large cohort of children born preterm, now aged 8-12 years. Preterm infants are a valuable group to study: firstly, because they provide an opportunity to distinguish between two factors important for later cholesterol metabolism, small size per se and prematurity (which may not have been adequately distinguished in some previously reported cohorts); and secondly, because they facilitate study of the potential impact on cholesterol metabolism of infant and childhood growth which is so variable in this cohort.

\section{Subjects and methods}

Children were originally recruited from a large cohort of infants $(n=926)$ who were enrolled in a randomised multicentre trial of nutrition in preterm infants. All weighed less than $1850 \mathrm{~g}$ at birth and were free from major congenital malformations or conditions likely to affect growth or neurodevelopment. They were recruited from five neonatal units (Cambridge, Ipswich, Kings Lynn, Norwich, and Sheffield) between 1982 and 1985. During their stay on the neonatal unit they were monitored by research nurses who performed regular anthropometry and recorded details of their progress, including clinical events and nutrition. Babies were randomly assigned to different diets (preterm formula, standard formula, donor breast milk) while in the neonatal unit; these diets are discussed in detail elsewhere ${ }^{18}$ However, there were no differences between diet groups in later cholesterol metabolism (unpublished results) and so for the purposes of this study all the dietary groups were combined. Details of social class, parity, and maternal health were collected at the time of enrolment in the trial. Surviving children were invited for follow up measurements at 18 months corrected age when their weight was recorded.

For the current follow up study, all surviving children still living in the UK were approached and invited to participate $(n=813)$. A total of $503(61.9 \%)$ agreed to do so and were seen by one of two investigators, either at their local hospital or general practitioner's surgery. A blood sample was obtained from 407 of the children. All were fasted for a minimum of six hours, and the time of sampling was recorded to the nearest 30 minutes. Samples were obtained between 9 am and 6 pm (median 12 $30 \mathrm{pm}$ ). Blood was obtained using a $23 \mathrm{~g}$ "butterfly" needle into lithium heparin bottles. Samples were kept on ice, separated within one hour, and plasma was frozen immediately at $-70^{\circ} \mathrm{C}$.

Daily fat intake from major sources was assessed by a dietary questionnaire adapted from the Health and Lifestyle survey. ${ }^{19}$ Detailed anthropometry was performed; weight measured to the nearest $10 \mathrm{~g}$ using digital electronic scales, height to the nearest $0.1 \mathrm{~cm}$ on a free standing mobile stadiometer, and skinfold thicknesses (biceps, triceps, subscapular, and suprailiac) using Harpenden skinfold calipers. A questionnaire was used to record details of current health and medications, and family history of ischaemic heart disease; parents were asked if any member of the family had/has high blood pressure, angina, heart attacks, or high blood cholesterol. The relationship of affected individuals to the subject was recorded as well as the age of onset, and, where relevant, age at death.

Plasma total cholesterol, high density lipoprotein (HDL), and triacylglycerol were determined by a method described previously ${ }^{4}$ using standard enzymatic methods. LDL cholesterol was estimated using Friedewald's formula. ${ }^{20}$ Apolipoprotein A1 and B were measured by an immunoturbidimetric method. ${ }^{21}$

Plasma total concentrations of lathosterol and campesterol were extracted and quantified by GC-MS ${ }^{16}$ with some modifications as follows. Deuterium labelled $\mathrm{D}_{5}$-lathosterol (internal standard) was added to plasma; the mixture was saponified by incubation with $50 \%$ potassium hydroxide and methanol, and then extracted with petroleum ether and silylated with $\mathrm{N}$-methyl-N-trimethylsilyltrifluoroacetamide. A $1 \mu \mathrm{l}$ aliquot of the derivatised extract was then injected into the Hewlett Packard 5970 GCMS. The intra-assay coefficient of variation for lathosterol at a concentration of $2 \mu \mathrm{mol} / 1$ was $7.6 \%$; for campesterol at $10 \mu \mathrm{mol} / 1$ it was $2.1 \%$. Results were expressed as $\mu \mathrm{mol} / 1$ of lathosterol and campesterol.

The campesterol concentrations were measured in 161 subjects only; however, the mean birth weight or plasma lathosterol were not significantly different in those with or without campesterol measurements $(p<0.4$ and $\mathrm{p}<0.3$ respectively).

\section{STATISTICAL METHODS}

The concentrations of plasma total, LDL, and HDL cholesterol and triacylglycerol were natural log transformed to normalise the data. Apolipoprotein A1, apolipoprotein B, and campesterol had normal distributions. Lathosterol was best normalised by transformation to its square root. For regression and correlation analysis transformed data were used. Data analysis was by SPSS.

Lathosterol and campesterol concentrations were corrected for plasma cholesterol concentration, by including cholesterol as an independent variable in the regression analysis. ${ }^{22}$ In addition the time at which the plasma sample was collected may affect lathosterol values because endogenous cholesterol synthesis has a diurnal rhythm in humans ${ }^{23}$; sample time was therefore also included in the regression analyses.

To compare children born at different gestational ages and seen at slightly different postnatal ages we used birth weight, weight at 18 months, and current weight and height standard deviation scores (SDS) which were derived from the UK growth reference data. ${ }^{24}$ The SDS indicates the number of standard deviations of a measurement from the mean for that age and sex. Current weight SDS and 
height SDS were used in combination to correct for current body size.

\section{Results}

Table 1 shows descriptive data for children and their current mean plasma lipids and noncholesterol sterols (lathosterol and campesterol). At 8-12 years of age, height and weight were similar in the two sexes but as expected females were significantly fatter than males. There were no significant differences in plasma lipids, lipoproteins, or non-cholesterol sterol concentrations between the sexes.

\section{RELATION BETWEEN SIZE AND CHOLESTEROL} METABOLISM

Multiple regression analysis was used to identify the relation between size or change in size and cholesterol metabolism at 8-12 years of age. The analyses were carried out separately for cholesterol, lathosterol, and campesterol as

Table 1 Age, anthropometry, and mean plasma cholesterol, lipids, and non-cholesterol sterol concentrations in the study population

\begin{tabular}{|c|c|c|c|c|c|c|}
\hline & \multicolumn{3}{|l|}{ Men } & \multicolumn{3}{|l|}{ Women } \\
\hline & $n$ & Mean & $S D$ & $n$ & Mean & $S D$ \\
\hline \multicolumn{7}{|l|}{ Birth measurements } \\
\hline Birth weight $(\mathrm{g})$ & 214 & 1402 & 280 & 197 & 1358 & 319 \\
\hline Birth weight SDS & 210 & -0.88 & 1.23 & 191 & -0.89 & 1.26 \\
\hline Birth length $(\mathrm{cm})$ & 128 & $41.1^{\star}$ & 2.62 & 136 & 40.4 & 3.20 \\
\hline Gestation (wk) & 214 & 31.03 & 2.70 & 196 & 31.12 & 2.76 \\
\hline Social class $\dagger$ & 206 & 3.49 & 1.29 & 189 & 3.54 & 1.35 \\
\hline \multicolumn{7}{|c|}{ Current measurements ( $8-12$ years old) } \\
\hline Age $(y)$ & 215 & 11.20 & 0.89 & 197 & 11.23 & 0.85 \\
\hline Weight $(\mathrm{kg})$ & 215 & 35.59 & 8.91 & 197 & 36.01 & 9.04 \\
\hline Weight SDS & 215 & -0.26 & 1.20 & 197 & -0.39 & 1.20 \\
\hline Height $(\mathrm{cm})$ & 215 & 142.01 & 9.00 & 197 & 142.02 & 9.10 \\
\hline Height SDS & 215 & -0.33 & 1.09 & 197 & -0.49 & 1.07 \\
\hline $\mathrm{BMI}\left(\mathrm{kg} / \mathrm{m}^{2}\right)$ & 215 & 17.31 & 3.02 & 197 & 17.60 & 2.89 \\
\hline BMI SDS & 215 & -0.13 & 1.22 & 197 & -0.24 & 1.17 \\
\hline Sum of 4 skinfolds (mm) & 213 & $34.21^{\star \star}$ & 16.04 & 196 & 40.30 & 15.57 \\
\hline \multicolumn{7}{|c|}{ Current plasma lipids and sterols } \\
\hline Total cholesterol $(\mathrm{mmol} / \mathrm{l})$ & 215 & 4.59 & 0.87 & 197 & 4.58 & 0.86 \\
\hline LDL cholesterol $(\mathrm{mmol} / \mathrm{l})$ & 198 & 2.79 & 0.78 & 176 & 2.80 & 0.67 \\
\hline HDL cholesterol $(\mathrm{mmol} / \mathrm{l})$ & 215 & 1.40 & 0.39 & 197 & 1.37 & 0.35 \\
\hline Triacylglycerol $(\mathrm{mmol} / \mathrm{l})$ & 215 & 0.87 & 0.53 & 196 & 0.92 & 0.43 \\
\hline Apolipoprotein A1 (g/l) & 2.07 & 1.57 & 0.26 & 1.93 & 1.55 & 0.25 \\
\hline Apolipoprotein B $(\mathrm{g} / \mathrm{l})$ & 2.14 & 1.01 & 0.21 & 1.96 & 1.02 & 0.24 \\
\hline Lathosterol $(\mu \mathrm{mol} / \mathrm{l})$ & 173 & 2.71 & 1.21 & 165 & 2.64 & 1.33 \\
\hline Campesterol $(\mu \mathrm{mol} / \mathrm{l})$ & 75 & 10.48 & 3.40 & 86 & 10.86 & 4.17 \\
\hline
\end{tabular}

Significantly different from women: ${ }^{\star} \mathrm{p}<0.05,{ }^{\star \star} \mathrm{p}<0.01$.

+Social class based on Registrar General coding ${ }^{36}$ using six categories, with class 3 manual and non-manual as separate categories and excluding non-classifiable cases.

Table 2 Factors independently predicting plasma cholesterol, lathosterol, and campesterol in children at 8-12 years of age born preterm

\begin{tabular}{|c|c|c|c|c|c|}
\hline Dependent variable & Independent variables & Coefficient & $S E$ & pvalue & $R^{2}$ \\
\hline \multirow[t]{2}{*}{ Cholesterol $\dagger$} & Current weight SDS & 0.031 & 0.012 & 0.008 & 0.04 \\
\hline & Current height SDS & -0.054 & 0.013 & $<0.001$ & \\
\hline Lathosterol $^{\star}$ & Birth weight SDS & -0.023 & 0.016 & 0.15 & 0.11 \\
\hline \multirow{2}{*}{ Lathosterol† } & Current weight SDS & 0.14 & 0.02 & $<0.001$ & 0.20 \\
\hline & Current height SDS & -0.07 & 0.02 & 0.003 & \\
\hline \multirow[t]{3}{*}{ Lathosterol $\ddagger$} & Birth weight SDS & -0.033 & 0.015 & 0.025 & 0.21 \\
\hline & Current weight SDS & 0.14 & 0.023 & $<0.001$ & \\
\hline & Current height SDS & -0.074 & 0.026 & 0.005 & \\
\hline Campesterol* & Birth weight SDS & 0.62 & 0.22 & 0.009 & 0.16 \\
\hline \multirow[t]{2}{*}{ Campesterol $\dagger$} & Current weight SDS & -1.43 & 0.31 & $<0.001$ & 0.24 \\
\hline & Current height SDS & 0.80 & 0.37 & 0.33 & \\
\hline \multirow[t]{3}{*}{ Campesterol $\ddagger$} & Birth weight SDS & 0.72 & 0.21 & 0.001 & 0.30 \\
\hline & Current weight SDS & -1.5 & 0.30 & $<0.001$ & \\
\hline & Current height SDS & 0.84 & 0.35 & 0.019 & \\
\hline
\end{tabular}

Sample time and plasma cholesterol were adjusted in all models of lathosterol and campesterol. Social class was adjusted for in all campesterol models.

Regression analysis with markers of cholesterol metabolism (plasma cholesterol, lathosterol, or campesterol) as dependent variable. Independent variables for each model were as follows: *birth size (birth weight SDS); †current size (current weight SDS, height SDS); †combined (birth weight SDS and current weight SDS and current height SDS).

$\mathrm{SE}$, standard error; $\mathrm{R}^{2}$, coefficient of determination. dependent variables. Each set of analyses tested the impact of: birth size; current size; both; and both plus their interaction. ${ }^{25}$

Birth size

Birth weight SDS was significantly and positively related to plasma campesterol concentrations $(p=0.009)$ but was not related to plasma lathosterol, total cholesterol, LDL cholesterol, HDL cholesterol, apolipoproteins, and triacylglycerol concentrations at 8-12 years of age (table 2).

\section{Current size}

Plasma total, LDL cholesterol, triacyglycerol, and lathosterol were significantly positively associated with current weight or weight SDS and negatively with height or height SDS. Plasma campesterol was significantly negatively related to weight and positively to height SDS. Plasma HDL cholesterol was significantly negatively related to weight SDS. The significance of weight and/or height disappeared when skinfold thickness was added to the regression, implying that current fatness is a better predictor of plasma total cholesterol, HDL cholesterol, lathosterol, and campesterol than current weight and/or height. Using either weight and height or skinfold thickness in the analysis did not alter the relation of lathosterol and campesterol with other independent variables in the regression. However, we chose to use weight and height SDS in the analyses because skinfold thickness was not measured at birth (table 2).

\section{Birth size and current size}

Birth weight SDS showed no association with plasma cholesterol, HDL cholesterol, apolipoprotein $\mathrm{A} 1$, apolipoprotein $\mathrm{B}$, or triacylglycerol, either before or after adjustment for current weight and height SDS. However, birth weight SDS was significantly positively related to campesterol with or without adjusting for current weight and height SDS. Plasma lathosterol was unrelated to birth weight SDS on its own, but significantly negatively related after adjusting for current weight and height SDS (table 2).

\section{Interaction}

There was no interaction between the effects of birth weight SDS and current weight SDS on plasma total cholesterol, LDL cholesterol, HDL cholesterol, apolipoprotein A1, apolipoprotein $\mathrm{B}$, or triacylglycerol, lathosterol, or campesterol - that is, a given change in size has the same impact irrespective of the child's birth weight.

\section{IMPACT OF SEX}

There were no interactions between sex and birth weight SDS or current weight SDS on plasma lathosterol or campesterol concentrations. This means that the relation between birth and current weight SDS with lathosterol and campesterol was similar in males and females. 
FURTHER ANALYSIS

Plasma campesterol was significantly positively related to social class. Increases in campesterol concentrations were associated with lower social class. However, including social class in the model with birth weight and weight and height SDS had no effect on the relation of variables with campesterol, except current height SDS which then became significant.

After adjusting for birth weight SDS, lathosterol and campesterol were not related to gestation, birth length, or ponderal index at birth and they did not remove the association with birth weight SDS.

To examine the influence of growth during different periods of infancy and childhood, measurements of growth during the early postnatal period (weight gain in hospital, weight at discharge) and weight in infancy (weight at 18 months) was included in the regression. None of these variables were related to later cholesterol metabolism parameters and they did not alter the relation with birth weight SDS.

Adjusting for age, pubertal status, mother's and father's height, and current fat intake from major sources made no appreciable difference to results.

\section{Discussion}

This study shows that plasma lathosterol and campesterol concentrations, indicators of endogenous cholesterol synthesis and absorption efficiency respectively, are related to early growth. Children born smaller for gestational age, at any gestation, had lower cholesterol absorption at 8-12 years of age, but it had no effect on their cholesterol synthesis. However, when current weight and height was adjusted for, the relation between birth weight and campesterol was strengthened and a relation with lathosterol revealed. Birth size adjusted for current size is a measure of change in size (centile crossing) between these two points (birth to 8-12 years). Thus among children of the same current size, predicted endogenous cholesterol synthesis was higher and cholesterol absorption efficiency lower in those whose weight centile had increased most between birth and current follow up. These data provide the first evidence that key components of cholesterol metabolism, absorption, and synthesis, may be influenced or programmed, not only by fetal growth (in the case of absorption) but also by growth in childhood.

We found no statistical interaction between birth weight for gestation and current weight for plasma lathosterol or campesterol. This means that a given change in fatness during childhood had the same impact on current lathosterol and campesterol irrespective of the child's birth weight for gestation. Thus the outcome changes in cholesterol metabolism we observed were seen not just in small babies, who have been emphasised as the vulnerable group in previous studies. ${ }^{10}$

An interaction between birth weight and later body mass index (BMI) on outcome was reported by Frankel et al who found that the increased risk of CVD associated with birth weight was restricted to obese adults. ${ }^{26}$ They concluded that CVD risk depends on a combination of early and later life factors. Leon and colleagues $^{27}$ and Lithell and colleagues ${ }^{28}$ reported similar interactions for blood pressure and non-insulin dependent diabetes respectively. While we did not find such an interaction, these studies and our own cohort concur in identifying postnatal anthropometry as being potentially influential for adult health. Nevertheless we found that neither growth in the first few weeks of life nor growth in the first 18 months were significantly related to campesterol or indeed lathosterol concentration at 8-12 years of age, and they did not change the influence of birth weight for gestation. This suggests that any influence of postnatal growth on cholesterol synthesis occurs between birth and 8-12 years.

In the current study plasma total, LDL, and HDL cholesterol, apolipoproteins, and triacylglycerol were not influenced by birth weight for gestation before or after correcting for current body weight. This may be explained by our finding that increased synthesis was compensated for by decreased absorption efficiency, hence plasma cholesterol homoeostasis was maintained. Leeson and colleagues ${ }^{29}$ also found no relation between plasma cholesterol and birth weight in term children 9-11 years old, yet endothelial function, an important early marker for atherosclerosis, was affected more in low birth weight children. Leeson et al did not however explore more detailed aspects of cholesterol metabolism. It remains to be seen whether the changes in cholesterol metabolism we report are associated with increased CVD risk even if, at this age, plasma cholesterol is unaffected.

Barker and colleagues found a higher incidence of death from coronary heart disease in men (birth between 1911 and 1930) born lighter compared to those born heavier, which could in part relate to early programmed changes in plasma lipids. ${ }^{30}$ It is possible that for the children in the current study, at 8-12 years of age, the effect of early growth on later plasma cholesterol concentration has yet to manifest itself. The modification of cholesterol metabolism detected in relation to size and growth in this study may be amplified throughout life, perhaps in response to ageing or interaction with other factors such as blood pressure and insulin resistance, such that frank changes in plasma lipids eventually occur. Follow up studies are needed to test this hypothesis.

Another factor to be considered when comparing our results with those of other investigators is that the children followed up in our study were born prematurely; the results cannot, therefore, be generalised to term children. Although gestational age (as opposed to size for gestational age) had no independent effect on either campesterol or lathosterol, our findings point to the need for similar studies in other population groups.

Previous investigators have found that whole body cholesterol synthesis, measured by the cholesterol balance method, is highly correlated with body weight. ${ }^{31}{ }^{32}$ In our study we found that plasma lathosterol concentration, a 


\section{Key messages}

- Reduced birth size for gestational age was associated with decreased cholesterol absorption efficiency at 8-12 years old children born preterm

- Greater catch up in weight between birth and $8-12$ years was strongly related to increased cholesterol synthesis and decreased absorption efficiency in all birth weight groups

- Current childhood fatness was related to increased cholesterol synthesis and decreased absorption efficiency even within the normal range of fatness

- Influence of pre- and postnatal growth on key markers of cholesterol metabolism during childhood may be important in adult cholesterol handling

marker for cholesterol synthesis, was also positively related to current body weight, even after adjusting for age and sex. Furthermore, our data showed that plasma campesterol was negatively related to body weight. This concurs with the previous observation that BMI was negatively associated with fractional cholesterol absorption as measured by a cholesterol tracer method in middle aged men. ${ }^{33}$ These concurrences add further weight to the validity of our outcome measures.

This study also shows for the first time evidence that current fatness (skinfold thickness) is associated with increased cholesterol synthesis (lathosterol) and decreased cholesterol absorption efficiency (campesterol). Nevertheless these preterm children were not fat as a group, with mean BMI SDS for boys -0.13 (SD 1.22) and for girls -0.24 (SD 1.17). This study therefore shows that even within a normal distribution of fatness, increasing fatness during childhood can affect later cholesterol metabolism.

A number of cell hypotheses have been proposed by Lucas $^{34}$ for the mechanism of molecular programming. Extending these concepts, Waterland and Garza ${ }^{35}$ suggested that hepatic polyploidisation (cells having more than the normal complement of chromosomes are called polyploid) which occurs during postnatal development may be permanently affected by factors in early life and consequently alter metabolism in adulthood. Hepatic polyploidisation may increase hepatic metabolic activity by enhancing enzyme gene expression over diploid hepatocytes. This could provide a possible basis for our findings as the liver is the prime site for cholesterol metabolism. However, future studies are required to provide information to support any suggested mechanisms which may be responsible for the biochemical associations observed in this study.

In conclusion, cholesterol absorption efficiency at the age of 8-12 years in children born preterm appears to be influenced by size at birth for gestation, postnatal growth, and current fatness. An increase in weight centile during childhood related to decreased cholesterol absorption efficiency, and increased synthesis. Although these changes in cholesterol metabolism were not reflected in the plasma cholesterol concentrations in the cohort, the longer term significance of our observation for CVD risk needs to be explored further.

We thank the staff of the neonatal unit in Norwich, Ipswich, Kings Lynn, Sheffield, and Cambridge for their collaboration; Drs Crowle, Bamford, Dossetor, and Pearse for their collaboration; the children taking part in the study and their families; and in particular Mr Baker for technical assistance. Contributors: MM carried out GC-MS analysis, analysed and interpreted the data, and wrote the paper; MF conducted the fieldwork, and contributed to writing, analysis; and interpretation of the data; TC gave statistical advice throughout the study and made key contributions to data interpretation and drafting of the paper; AI initiated and designed the original study and made AL initiated and designed the original study and made study. Funding was by the Medical Research Council.

1 Keys A. Seven countries; a multivariate analysis on death and Keys A. Seven countries; a multivariate analysis on death and coronary heart diseas

2 Kannel WB, Neaton JD, Wentworth D, et al. Over all coronary heart disease mortality rates in relation to major risk factors in 325,348 men screened for the MRFIT. Am Heart f 1986;112:4825-37.

3 Hahn P. Effect of litter size on plasma cholesterol and insuin and some liver and adipose tissue enzymes in adult rodents. F Nutr 1984;114:1231-4.

4 Lucas A, Baker BA, Desai M, Hales CN. Nutrition in pregnant or lactating rats programs lipid metabolism in the offspring. Br f Nutr 1996;76:605-12.

5 Mortaz M, Cole TJ, Lucas A. Early maternal dietary protein manipulation and adult offspring cholesterol metabolism. Proc Nutr Soc 1999 (in press) 1999;58:53A.

6 Mott GE, Jackson EM, McMahan CA, McGill HC. CholesMott GE, Jackson EM, McMahan CA, McGill HC. Choles-
terol metabolism in adult baboons is influenced by infant terol metabolism in adult bab

7 Fall CHD, Barker DJP, Osmond C, Winter PD, Clark PMS, Hales CN. Relation of infant feeding to adult serum cholesterol concentration and death from ischemic heart disease. BMF 1992;304:801-5

8 Barker DJP, Meade TW, Fall CHD, et al. Relation of fetal and infant growth to plasma fibrinogen and factor VII concentrations in adult life. BMF 1992;304:148-52.

9 Hales CN, Barker DJP, Clark PMS, et al. Fetal and infant growth and impaired glucose tolerance at age 64 . BMf 1991;303:1019-22.

10 Barker DJP, Martyn CN, Osmond C, Hales CN, Fall CHD. Growth in utero and serum cholesterol concentrations in adult life. BMF 1993;307:1524-7.

11 Eriksson JG, Forsen T, Tuomilehto J, Winter PD, Osmond C, Barker DJP. Catch up growth in childhood and death from coronary heart disease; longitudinal study. BMF 1999;318:427-31

12 Stary HC. Evolution and progression of atherosclerotic lesions in coronary arteries of children and young adults. Arteriosclerosis 1989;9:19-32.

13 Tilvis RS, Miettinene TA. Serum plant sterols and their relation to cholesterol absorption. Am f Clin Nutr 1986;43: 92-7.

14 Bjorkhem I, Miettinen TA, Reihner E, Ewerth S, Angelin B. Correlation between serum levels of some cholesterol precursors and activity of HMG CoA reductase in human liver. F Lipid Res 1987;28:1137-43.

15 Kempen HJM, Glatz JFC, Gevers Leuven JA, van der Voort HA, Katan MB. Serum lathosterol concentration is an indicator of whole-body cholesterol synthesis in humans. 7 Lipid Res 1988;29:1149-55

16 Farkkila MA, Tilvis RS, Miettinen TA. Regulation of plasma plant sterol levels in patients with gut resections plasma plant sterol levels in patients with

17 Cox CMA, Sutherland WHF, Ball MJ, Mann JI. Response of plasma lathosterol concentration to change in the quality of dietary fat in men and women. Eur 7 Clin Nutr 1996; ity of dietary

18 Lucas A, Gore SM, Cole TJ, et al. Multicentre trial on feeding low birth weight infants: effect of diet on early growth. Arch Dis Child 1984;59:722-30.

19 Cox BD, Blaxter M, Buckle ALJ et al, eds. The health and lifestyle survey. London: Health Promotion Research Trust, 1987.

20 Friedewald WT, Levy RI, Fredrickson DS. Estimation of concentration of low-density lipoprotein cholesterol in plasma, without use of preparative ultracentrifuge. Clin Chem 1972;18:499-502.

21 Becker W, Rapp W, Schenk HG, Storiko K. Method for the quantitative determination of plasma proteins by immunopercipitation. Z Klin Chem Klin Biochem 1968;6:113-22.

22 Miettinen TA, Tilvis RS, Kesaniemi YA. Serum plant sterol and cholesterol precursors reflect cholesterol absorption and synthesis in volunteers of a randomly selected male and synthesis in volunteers of a randomly
population. Am f Epidemiol 1990;131:20-31. 
23 Jones PJH, Schoeller DA. Evidence of diurnal periodicity in human cholesterol synthesis. F Lipid Res 1990;31:667-73.

24 Freeman JV, Cole TJ, Chinn S, Jones PRM, White EM, Preece MA. Cross-sectional stature and weight reference curves for the UK. Arch Dis Child 1995;73:17-24

25 Lucas A, Fewtrell MS, Cole TJ. The "fetal origin of adult diseases" hypothesis revisited. BMF 1999;319:245-9

26 Frankel S, Elwood P, Sweetnam P, Yarnell J, Smith GD. Birth weight, body mass index in middle age and incidence of coronary heart disease. Lancet 1996;348:1478-80.

27 Leon DA, Koupilova I, Lithell HO, et al. Failure to realise growth potential in utero and adult obesity in relation to blood pressure in 50 year old Swedish men. $B M^{\mathcal{F}}$ 1996;312:401-6.

28 Lithell HO, McKeigue PM, Berglund L, Mohsen R, Lithell $\mathrm{U}$, Leon DA. Relation of size at birth to non-insulin dependent diabetes and insulin concentrations in men aged 50-60 years. BMF 1996;312:406-10.

29 Leeson CPM, Whincup PH, Cook DG, Donald AE, Papacosta O, Lucas A, Deanfield JE. Circulation 1997;96: 2233-8.
30 Barker DJP, Osmond C, Winter PD, Margetts B, Simmonds SJ. Weight in infancy and death from ischemic heart disease. Lancet 1989;2:577-80.

31 Nestel PG, Whyte HM, Goodman DS. Distribution and turnover of cholesterol in humans. $\mathcal{F}$ Clin Invest 1969;48: 982-91

32 Goodman DS, Smith FR, Seplowitze AH, et al. Prediction of the parameters of whole body cholesterol metabolism in humans. F Lipid Res 1980;21:699-713.

33 Miettienen TA, Kesaniemi YA. Cholesterol absorption: regulation of cholesterol synthesis and elimination and within population variations of serum cholesterol levels. Am f Clin Nutr 1989;19:629-35.

34 Lucas A. Programming by early nutrition: an experimental approach. F Nutr 1998;128:401S-406S.

35 Waterland RA, Garza C. Potential mechanisms of metabolic imprinting that lead to chronic disease. Am $\mathcal{F}$ Clin Nutr 1999;69:179-97.

36 Morley R, Cole TI, Powell R, Lucas A. Mothers choice to provide breast milk and developmental outcome. Arch Dis Child 1988;63:1382-5.

\section{Rapid responses}

Letters on the following papers have been published recently as rapid responses on the $A D C$ website. To read these letters visit www.archdischild.com and click on "Read rapid responses":

Do presenters to paediatric meetings get their work published? F A I Riordan. Arch Dis Child 2000;83:524-6.

A case of lead poisoning due to snooker chalk. P I Dargan, P H Evans, I M House, et al. Arch Dis Child 2000;83:519-20.

The management of fever and petechiae: making sense of rash decisions. P A Brogan, A Raffles. Arch Dis Child 2000;83:506-7.

Sudden unexpected non-violent death between 1 and 19 years in north Spain. B Morentin, B Aguilera, P M Garamendi, et al. Arch Dis Child 2000;82:456-61.

Emergency management of meningococcal disease. A J Pollard, J Britto, S Nadel, et al. Arch Dis Child 1999;80:290-6.

Parents' perceptions of disclosure of the diagnosis of cerebral palsy. G Baird, H McConachie, D Scrutton. Arch Dis Child 2000;83:475-80.

The need for and the role of a coordinator in child health surveillance/promotion. M Blair. Arch Dis Child 2001;84:1-5.

Case-control study of current validity of previously described risk factors for SIDS in the Netherlands. M P l'Hoir, A C Engelberts, G Th J van Well, et al. Arch Dis Child 1998;79:386-93.

If you would like to post an electronic response to these or any other articles published in the journal, please go to the website, access the article in which you are interested, and click on "eLetters: Submit a reponse to this article" in the box in the top right hand corner. 